\title{
Representative Solutions for Bi-Objective Optimisation
}

\author{
Emir Demirović \\ School of Computing and Information Systems \\ University of Melbourne \\ Melbourne, Australia \\ emir.demirovic@unimelb.edu.au
}

\author{
Nicolas Schwind \\ National Institute of Advanced \\ Industrial Science and Technology \\ Tokyo, Japan \\ nicolas-schwind@aist.go.jp
}

\begin{abstract}
Bi-objective optimisation aims to optimise two generally competing objective functions. Typically, it consists in computing the set of nondominated solutions, called the Pareto front. This raises two issues: 1) time complexity, as the Pareto front in general can be infinite for continuous problems and exponentially large for discrete problems, and 2) lack of decisiveness. This paper focusses on the computation of a small, "relevant" subset of the Pareto front called the representative set, which provides meaningful trade-offs between the two objectives. We introduce a procedure which, given a precomputed Pareto front, computes a representative set in polynomial time, and then we show how to adapt it to the case where the Pareto front is not provided. This has three important consequences for computing the representative set: 1) does not require the whole Pareto front to be provided explicitly, 2) can be done in polynomial time for bi-objective mixed-integer linear programs, and 3) only requires a polynomial number of solver calls for bi-objective problems, as opposed to the case where a higher number of objectives is involved. We implement our algorithm and empirically illustrate the efficiency on two families of benchmarks.
\end{abstract}

\section{Introduction}

Bi-objective optimisation aims to optimise two competing objective functions. For instance, in supply-chain management, the goal is to simultaneously minimise delivery time and delivery cost (Trisna et al. 2016). In the design of wireless sensor networks, both energy consumption and data collection time must be kept at a minimum (Caillouet, $\mathrm{Li}$, and Razafindralambo 2011). An "ideal" option generally does not exist and trade-offs must be made.

The set of nondominated solutions, called the Pareto front, is exponentially large in the general case. This brings forth two main issues. First, enumerating the Pareto front can be computationally infeasible. Second, providing a large number of options might hinder decision making, as providing an overwhelming number of choices can be counterproductive (Iyengar and Lepper 2000; Shafir, Simonson, and Tversky 1993; Dhar 1997).

Copyright (C) 2020, Association for the Advancement of Artificial Intelligence (www.aaai.org). All rights reserved.

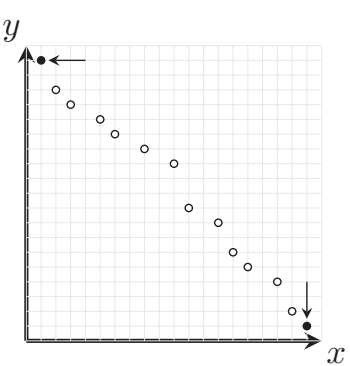

(a) Diverse Solutions

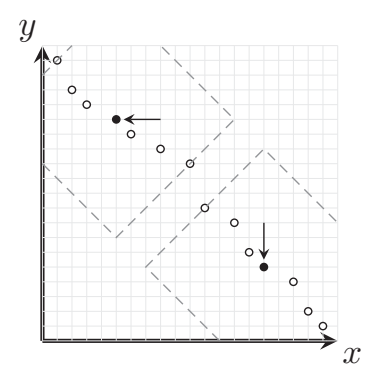

(b) Representative Solutions.
Figure 1: Two notions of Pareto front filtering when two solutions are to be selected in each case. The dashed lines illustrate the radius of the corresponding representative set

A reasonable alternative is then to consider a subset of the Pareto front. Two main notions of such Pareto front filtering have been introduced in the literature. Both notions depend on a number $k$ which is assumed to be "small" and given in input of the problem. The first one, called $d i$ versity (or uniformity) (Sayin 2000; Hebrard et al. 2005; Petit and Trapp 2015; Vaz et al. 2015; Masin and Bukchin 2008), aims to select $k$ solutions from the Pareto front that are "spread out" as much as possible. The second one, called representativity (or coverage) (Sayin 2000; Vaz et al. 2015; Schwind et al. 2016), considers a set $S$ of $k$ solutions from the Pareto front with minimum radius, where the radius of $S$ is the maximal distance between any solution $p$ from the Pareto front and the solution from $S$ that is the closest to $p$; such a set $S$ is called a representative set (cf. Fig. 1).

In this paper, we focus on the computation of a representative set of solutions in the bi-objective case. In particular, we pose the following research questions:

1. Is it possible to efficiently compute a representative set without computing the whole complete Pareto front?

2. Can a representative set be computed even if the Pareto front is infinite?

Our contribution provides a positive answer to both questions. This is done constructively: we provide a generic procedure for bi-objective optimisation that computes a rep- 
resentative set using only a polynomial number of solver calls. The notion of a solver call depends on the structure of the considered optimisation problem and refers to either a sub-procedure that solves the single-objective version of the problem, e.g. interior-point method for linear programs, or an oracle for problems whose decision counterpart is NPcomplete. In general, the Pareto front is infinite for linear programs and exponentially large for discrete optimisation problems. Considering only a polynomial number of solver calls is a clear advantage over enumerating the Pareto front when computing a representative set. As a proof-of-concept, we implement our procedure and empirically show that it computes the representative set in reasonable time.

In the next section, we position our paper with respect to related work and provide further motivation. We then follow up with preliminaries and our main contributions.

\section{Related Work}

We assume that the reader is familiar with the complexity classes P and NP (see (Papadimitriou 2003) for more details). Higher complexity classes are defined using oracles, i.e. abstract machines that solve problems from a particular class in constant time. In particular, $\mathrm{P}^{\mathrm{NP}}$ (resp. NP $\mathrm{PP}^{\mathrm{N}}$ ) is the class of decision problems that are solved in polynomial time by a deterministic (resp. non-deterministic) Turing machine using an oracle for NP.

In (Schwind et al. 2016), the authors introduced the notion of representative solutions for multi-objective constraint optimisation problems. They proved that the decision problem underlying the computation of a representative set of $k$ solutions ( $k$ being given in unary form) is NP ${ }^{N P}$-hard in the general multi-objective case. They also introduced a procedure that first enumerates the Pareto front, then solves the NPhard task of computing a represented set of $k$ solutions. Our aim is to show the computational advantages of focussing on only two objectives. This is motivated by the fact that when the Pareto front is given in input, computing a representative set is an NP-hard task in the general multi-objective case (Schwind et al. 2016), but can be done in polynomial time in the bi-objective case. Our goal is then to investigate whether the restriction to two objectives also leads to a computational shift in the case when the Pareto front is not explicitly given. As presented in the following sections, this is indeed the case.

To the best of our knowledge, computing a representative set for the dedicated bi-objective case has not been addressed in the literature. Instead, the research efforts have been focussed on developing heuristics to compute (not necessarily representative) subsets of the Pareto front for biand multi-objective problems. In this context, heuristic approaches compute (Pareto optimal) solutions that aim to cover the Pareto front, but do not guarantee the optimality of the set with respect to the desired filtering notion.

There is a number of works aiming to filter the Pareto front by selecting only a few Pareto optimal solutions and obtain a "good" representation (Eusébio, Figueira, and Ehrgott 2014; Vaz et al. 2015; Schwind et al. 2016; Raith and Sedeño-Noda 2017; Masin and Bukchin 2008;
Ceyhan, Köksalan, and Lokman 2019). How well a Pareto front is represented by means of a few solutions depends on the context. As mentioned in the introduction, there are two main such quality measures (Fig. 1b): diversity (or uniformity) and representativity (or coverage). There is also a third measure called $\epsilon$-indicator considered in (Zitzler et al. 2003; Vaz et al. 2015), which is closely related to the one of representativity. However, this measure consists in computing a restricted set of solutions that are "close enough" to the Pareto front, but that are not necessarily Pareto optimal. An orthogonal diversity measure can be considered over decision variables rather than objectives, i.e., computing nearoptimal solutions with a structure of diverse nature (Adomavicius and Kwon 2014). The authors in (Petit and Trapp 2019) provide a detailed survey on related diversity measures. One of the motivations for diversity is that it might not be easy to capture the true preferences of users in the form of an objective function, and thus providing a variety of solutions, which can be further filtered offline and online, is beneficial. In our work, in contrast, we assume that qualitative measures are possible for two objectives, for instance production time versus cost. In these cases, the objectives functions can be used to succintly explain the differences among solutions and their trade-offs to the user. Note that a discrimination based on both decision variables and objectives is not incompatible. Indeed, one could consider computing a representative set, which provides meaningful trade-offs between two objectives, and afterwards search for diverse solutions among those, in an attempt to capture other important solution features that were not given explicitly.

In (Bazgan, Jamain, and Vanderpooten 2017), the authors introduce the notion of $\epsilon$-Pareto front $(\epsilon-\mathrm{PF})$, which is a set of solutions that dominate each Pareto optimal solution in an $\epsilon$-relaxed sense. The authors discuss algorithms for generating the smallest $\epsilon$-PF with additional properties and variations. This notion is related but is not directly comparable to the representative set, as the goals are different: it aims to approximate the Pareto front rather than compute a representation with a few solutions.

Focussing on the bi-objective optimisation, network flow problems are solved in (Eusébio, Figueira, and Ehrgott 2014; Raith and Sedeño-Noda 2017) by computing only a subset of the Pareto front. However, the quality measure is again different from the representativity measure. In (Raith and Sedeño-Noda 2017), so-called "extreme" solutions are selected, i.e. the Pareto optimal solutions that lie on the boundaries of the convex hull of the solution space. In (Eusébio, Figueira, and Ehrgott 2014), the notion of representativity is the same as the one considered here, but only heuristics are provided: a subset of the Pareto front is computed in an online fashion, and does not necessarily result in a representative set. The number of returned solutions is not decided as an upstream step; instead, the user is required to provide the desired distance betweens solutions in a semiinteractive process. For representative solutions of multiobjective problems with continuous variables, the authors in (Karasakal and Köksalan 2009) propose a heuristic to sample a surface that approximates the Pareto front.

Closely related to our work is (Vaz et al. 2015): the au- 
thors introduce two complete algorithms to compute a representative set of $k$ solutions for bi-objective optimization problems, under the assumption that the Pareto front is available in input. Their most efficient algorithm is based on Dynamic Programming and runs in $\mathcal{O}(k \cdot|P F|+|P F|$. $\log |P F|)$, where $|P F|$ is the size of the Pareto front. Similarly to our procedure presented in the next section, the first step is to re-order the Pareto front in an increasing order according to their values projected to first objective. Then, roughly speaking, their algorithm iteratively computes the radius of a representative set of size $k^{\prime}$ where $k^{\prime}$ ranges over $\{1, \ldots, k\}$. This departs from our method which, in a nutshell, consists in computing a representative set of a minimum number of $k^{\prime}$ solutions given a fixed radius $R$, and doing so iteratively by performing a dichotomic search over the radius values ranging between two bounds until some optimality conditions are met and $k^{\prime}$ coincides with the value $k$ required in input. Most importantly, in (Vaz et al. 2015) the authors focussed on the case where the Pareto front is given in input, and did not consider the case where it is characterised succinctly, e.g. by means of constraints.

Two constraint programming approaches have been proposed for multi-objective optimisation. In (Gavanelli 2002), the author introduces a data structure for storing the Pareto front. This is used in an anytime depth-first search algorithm that eventually enumerates the whole Pareto front. The approach has been refined into a so-called global constraint (Schaus and Hartert 2013), which is suitable for constraint programming solvers, and used in a large neighbourhood search framework. The idea is to maintain a list of nondominated solutions and to iteratively select one solution from the set, relax and optimise it so as to improve the hypervolume surrounding the maintained nondominated set of solutions. While related, the nature of our paper is very different to (Schaus and Hartert 2013): they consider a heuristic to approximate the Pareto front with many solutions, while we consider a complete algorithm for representing the Pareto front with few solutions.

The most popular algorithms for enumerating bi-objective Pareto frontiers are the $\epsilon$-method (Haimes 1971) and the two-phase approach (Ulungu and Teghem 1995). The $\epsilon$ method systematically enumerates the complete Pareto front for bi-objective problems: it computes an initial solution by lexicographically minimising $f_{x}$ and $f_{y}$ and iteratively computes lexicographical solutions that minimise the first and then the second objective function while requiring the value according to the first objective function to be greater than previously computed. In the two-phase algorithm, the decision variables are assumed to be binary. It consists of two steps: 1) to compute the nondominated solutions that lie on the convex hull of the Pareto front, and 2) to compute the missing solutions by considering the space in between two consecutive nondominated solutions of the convex hull. The first stage is generic, but the second one is problem-specific. Indeed, in our experiments, the $\epsilon$-method gave better results than the two-phase approach with a generic second-stage implementation. A refinement of the two-phase approach (Stidsen, Andersen, and Dammann 2014) may have a better performance depending on the problem. We refer to (Stid- sen, Andersen, and Dammann 2014) for a survey of others techniques and references for problem-specific variants.

In (Bergman and Cire 2016), a binary-decision diagram (BDD) approach is applied to compute the Pareto front for multi-objective optimisation. The strength of the method is that once a combinatorial problem is represented as a BDD, a multi-objective shortest path algorithm can be used to compute an optimal solution. The main drawback is that not all problems admit a compact BDD representation.

\section{Preliminaries}

We are given a fixed set $V$ of integer variables and a set of constraints $C$. An assignment of all variables $V$ to a value is called a solution if it satisfies all constraints from $C$. We assume that checking whether a given assignment is a solution can be done in polynomial time. For simplicity, we use $C^{*}$ as the (succintly characterised) set of solutions. We are also given two objective functions $f_{x}, f_{y}$ of the form $f: C^{*} \rightarrow \mathbb{Q}$, which are to be minimized simultaneously. A solution $p \in C^{*}$ dominates another solution $p^{\prime} \in C^{*}$ whenever $f_{x}(p) \leqslant f_{x}\left(p^{\prime}\right)$ and $f_{y}(p) \leqslant f_{y}\left(p^{\prime}\right)$. We say that $p$ strictly dominates $p^{\prime}$ if $p$ dominates $p^{\prime}$ and $p^{\prime}$ does not dominate $p$. And $p$ is said to be Pareto optimal if there is no solution $p^{\prime}$ that strictly dominates $p$. The set $P F$ denotes the Pareto front, i.e., the set of all Pareto optimal solutions.

The Manhattan distance (or $L 1$-norm) between two solutions $p, p^{\prime} \in C^{*}$ is defined as $\operatorname{dist}\left(p, p^{\prime}\right)=\left|f_{x}(p)-f_{x}\left(p^{\prime}\right)\right|+$ $\left|f_{y}(p)-f_{y}\left(p^{\prime}\right)\right|$. In the following, we focus on the Manhattan distance and simply refer to it as the "distance".

The radius of a set of Pareto optimal solutions $S \subseteq P F$, denoted as $\Omega(S)$, is defined as

$$
\Omega(S)=\max _{p^{\prime} \in P F} \min _{p \in S} \operatorname{dist}\left(p, p^{\prime}\right) .
$$

We say that a set $S \subseteq P F$ is optimally representative of $P F$ (representative for short) if $S$ has a minimal radius among all sets $S^{\prime} \subseteq P F$ such that $\left|S^{\prime}\right|=|S|$. When $|S|=k$ and $S$ is a representative set, we also say that $S$ is a $k$ representative set. Intuitively, a representative set $S$ is such that every Pareto optimal solution is as close as possible to some solution of $S$. Our ultimate goal is given a bi-objective optimisation problem and positive integer $k$, to find a $k$ representative set of solutions.

\section{Computing a Representative Set When the Pareto Front is Available}

We introduce a procedure for computing a $k$-representative set of solutions, given $k$ and assuming that the Pareto front is also given e.g. when it has been computed as an upstream step. The advantage of our procedure is that it can then be adapted to the case where the Pareto front is not given explicitly but by means of a set of variables $V$ and a set of constraints $C$. This will be shown in the next section, roughly speaking, by substituting some of the operations with solver calls. The signature of our procedure is as follows:

Definition $1(\operatorname{RepSet}(P F, k))$

- Input: Pareto front PF, integer $k$. 


\section{- Output: A k-representative set of solutions.}

The key step of our procedure is, given a fixed radius $R$, to compute a $k^{\prime}$-representative set $S$ such that $\Omega(S) \leqslant R$ while minimizing $k^{\prime}$. This step is denoted hereafter by $R S$ $R(P F, R)$, where $R$ is a radius and $P F$ is a sorted Pareto front, i.e. a Pareto front where all solutions are sorted in a non-decreasing order according to $f_{x}$.

The following helper methods are used in $R S-R(P F, R)$ :

- furthest_within_radius $(p, P F, R)$, or $f w r(p, P F, R)$ for short: given a Pareto front $P F$, a solution $p \in P F$, and a radius $R$, it is defined as

$$
f w r(p, P F, R)=\underset{p^{\prime} \in P F}{\arg \max }\left\{f_{x}\left(p^{\prime}\right) \mid \operatorname{dist}\left(p, p^{\prime}\right) \leqslant R\right\} .
$$

Note that the method is guaranteed to produce a solution: the reference solution $p$ can be returned.

- $\operatorname{filter}(p, P F, R)$ : given a Pareto front $P F$, a solution $p \in$ $P F$ and a radius $R$, it computes a new Pareto front $P F^{\prime}$, which contains solutions from $P F$ that are distant from $p$ by at least $R$, i.e. it is defined as

$$
\text { filter }(p, P F, R)=\left\{p^{\prime} \mid p^{\prime} \in P F \wedge \operatorname{dist}\left(p, p^{\prime}\right)>R\right\} .
$$

- $p_{\min }^{x}(P F)$ : given a Pareto front $P F$, it is defined as

$$
p_{\text {min }}^{x}(P F)=\underset{p \in P F}{\arg \min }\left\{f_{x}(p)\right\} .
$$

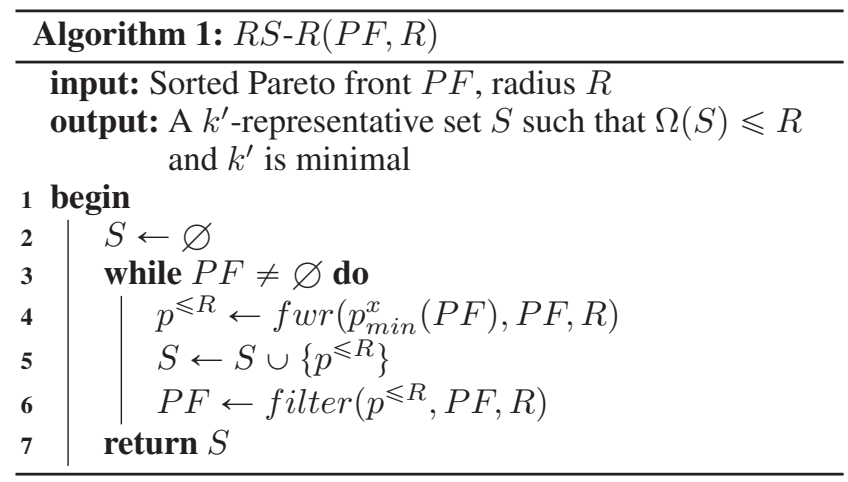

The method $R S-R(P F, R)$ is described in Algorithm 1. The representative set $S$ is initially set to empty (line 2). The first solution $p^{\leqslant R}$ to be added to $S$ is defined as the most distant solution from the leftmost solution $p_{\text {min }}^{x}(P F)$ within the distance range $R$ (lines 4 and 5). Then all solutions within the range of $R$ from the selected solution $p^{\leqslant R}$ are removed from the Pareto front (line 6), and the process is repeated until the Pareto front becomes empty. The method is illustrated in Fig. 2 (a-c) through an example. In the figures, $p^{\leqslant R}$ and $p_{\min }^{x}$ denote respectively the solutions $p^{\leqslant R}$ and $p_{\min }^{x}(P F)$ at the corresponding step (lines 4 and 5 in Algorithm 1), i.e. when $P F$ is updated accordingly (line 6 from the previous iteration in Algorithm 1).

Proposition 1 Algorithm 1 is correct, i.e. it computes a $k^{\prime}$ representative set $R S-R(P F, R)$ of solutions $S$ such that $\Omega(S) \leqslant R$ and $k^{\prime}$ is minimal.

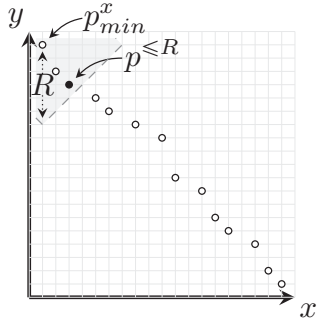

(a) Initial step

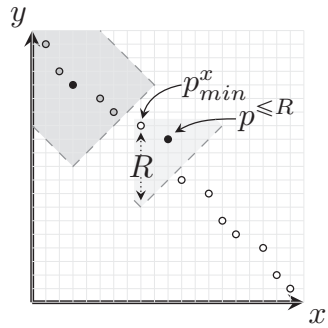

(b) Second step

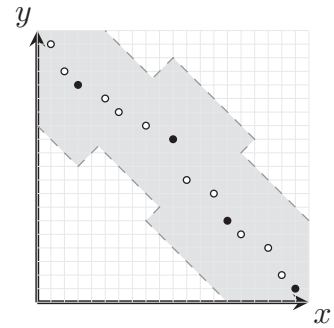

(c) Last step
Figure 2: Illustration of Algorithm 1

Proposition 2 Algorithm 1 runs in $\left.k^{\prime} \cdot \log (|P F|)\right)$ time, where $k^{\prime}$ is the cardinality of the smallest representative set $S$ with $\Omega(S) \leqslant R$.

We are now ready to describe our main procedure $\operatorname{RepSet}(P F, k)$, which uses the method $R S-R(P F, R)$ iteratively by performing a dichotomic search on the radius between two bounds. At each such iteration step, 1) the radius $R$ is increased or decreased depending on whether the value $k^{\prime}$ resulting from the computation in the previous step is lower or greater than the required number $k$; and $2)$ the two bounds are updated accordingly. The procedure $\operatorname{RepSet}(P F, k)$ terminates once the two bounds meet.

The following helper methods are used:

- $p_{\max }^{x}(P F)$ : given a Pareto front $P F$, it is defined as

$$
p_{\text {max }}^{x}(P F)=\underset{p \in P F}{\arg \max }\left\{f_{x}(p)\right\} .
$$

- $\operatorname{sort}(P F)$ : given a Pareto front $P F$, it sorts the solutions from $P F$ in a non-decreasing order according to $f_{x}$.

The procedure RepSet $(P F, k)$ is described in Algorithm 2. We assume integer-valued objectives. Initially, the Pareto front is sorted in a non-decreasing order according to $f_{x}$ (line 2). This step allows one to reduce the time complexity of the subsequent methods. The bounds are initially set in lines 3 and 4 for the purpose of efficiency, i.e. in such a way that the initial radius defined in line 7 implies the representative set computed at the first iteration step (line 8) to contain at most $k$ solutions. Given these bounds, the algorithm performs a classical dichotomic search.

Proposition 3 Algorithm 2 is correct, i.e. it computes a $k$ representative set RepSet $(P F, k)$.

Proposition 4 Algorithm 2 runs in $\mathcal{O}(k \cdot \log (|P F|) \cdot$ $\left.\log \left(R_{U B}\right)+|P F| \cdot \log (|P F|)\right)$, where $R_{U B}=$ $\left\lfloor\frac{\operatorname{dist}\left(p_{\min }^{x}, p_{\max }^{x}\right)}{k}\right\rfloor$. 


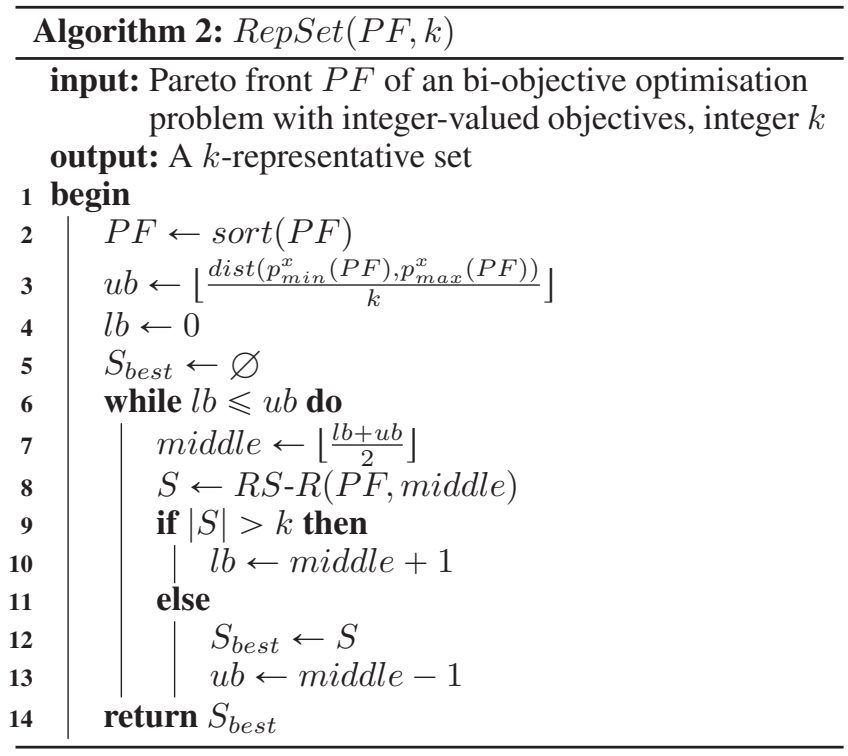

For rational objective functions, the algorithm needs to be modified to use dichotomic search defined on rational numbers (Kwek and Mehlhorn 2003). In this case, the complexity would substitute $R_{U B}$ with $\max (p, q)$, where $p$ and $q$ are the maximum denominator and numerator out of all considered rational numbers. Alternatively, the floor functions can be removed from lines 3 and 7, an up-to-precision small $\epsilon>0$ can be used instead of the constant 1 in lines 10 and 13 , and the procedure could stop once the lower and upper bounds are within a predefined distance (line 6).

As in (Vaz et al. 2015), the obtained complexity is dominated by the complexity of sorting. The advantage of our algorithm, however, is that it can be extended to the implicitlydefined Pareto front, as shown in the next section.

\section{Computing a Representative Set in Constraint-Based Bi-Objective Optimisation}

We adapt the procedure $R S-R(P F, R)$ presented in the previous section to the case when the Pareto front is not available, but instead succinctly characterised by means of a biobjective optimisation problem. The adapted procedure, denoted now by $R S-R 2\left(\left\langle X, C, f_{x}, f_{y}\right\rangle, R\right)$, follows the same scheme as presented in Algorithm 1, but the helper functions fwr, filter, and $p_{\text {min }}^{x}$ used in defining the method $R S-R$ must be updated since the Pareto front is no longer available. The following helper methods are used in $R S$ $R_{2}\left(\left\langle X, C, f_{x}, f_{y}\right\rangle, R\right)$. These methods are similar as presented in the previous section, but updated to new signatures.

Helper method $f w r 2\left(p^{r e f},\left\langle X, C, f_{x}, f_{y}\right\rangle, R\right)$ : Given a Pareto optimal solution $p^{r e f}=\left(p_{x}^{r e f}, p_{y}^{r e f}\right)$, a bi-objective optimisation problem $\left\langle X, C, f_{x}, f_{y}\right\rangle$, and a radius $R$, the task is to compute the Pareto optimal solution $p^{\leqslant R}$ :

$$
p^{\leqslant R}=\underset{p^{\prime} \in P F}{\arg \max }\left\{f_{x}\left(p^{\prime}\right) \mid \operatorname{dist}\left(p^{r e f}, p^{\prime}\right) \leqslant R\right\} .
$$

The Pareto front $P F$ is not given. We do not need to compute it entirely, but rather solve a series of optimisation problems. Prior to this, let us discuss necessary subproblems.

To compute $p^{\leqslant R}$, we only need to consider as candidates the solutions $p^{\prime}$ that are not dominated by other solutions $p$ such that $\operatorname{dist}\left(p^{r e f}, p\right)>R$. Ensuring Pareto optimality is one of the main challenges. This is done by initially computing a bound for the second objective $f_{y}$ for Pareto optimal solutions $p^{\prime}$ such that $\operatorname{dist}\left(p^{r e f}, p^{\prime}\right) \leqslant R$. The bound is used to compute the Pareto optimal solution $p^{>R}$ that is the closest one to $p^{\leqslant R}$ but outside the range of $p^{\text {ref }}$ w.r.t. the radius $R$. Lastly, we compute the solution $p^{\leqslant R}$ by searching for the Pareto optimal solution that is maximal w.r.t. $f_{x}$ while remaining within the range of $p^{r e f}$ w.r.t. the radius $R$ and nondominated by $p^{>R}$. The bound is computed as:

$$
\begin{gathered}
y_{\text {bound }}=\min _{p \in C^{*}} f_{y}(p) \\
p_{y}^{r e f}-f_{y}(p)+f_{x}(p)-p_{x}^{r e f} \leqslant R
\end{gathered}
$$

Eq. 2 constrains the solution to be within the range of $p^{\text {ref }}$ w.r.t. the radius $R$. Please note that the distance is defined as a norm, but we simplified the equation by taking into account that solutions are computed in increasing $f_{x}$ value. The Pareto optimal solution $p^{>R}$ that is the closest one to $p^{\leqslant R}$ but outside the range of $p^{\leqslant R}$ w.r.t. the radius $R$, is computed using lexicographical optimisation:

$$
\begin{gathered}
p^{>R}=\underset{p \in C^{*}}{\arg \min } f_{x}(p): \underset{p \in C^{*}}{\arg \min } f_{y}(p) \\
p_{y}^{r e f}-f_{y}(p)+f_{x}(p)-p_{x}^{r e f}>R \\
f_{y}(p)<y_{\text {bound }}
\end{gathered}
$$

Please note that the solution $p^{>R}$ is Pareto optimal, since we have that $p_{y}^{>R}<y_{\text {bound }}$ and $p^{>R}$ is minimised. The last step is to compute the Pareto optimal solution $p^{\leqslant R}$ using lexicographical optimisation:

$$
\begin{gathered}
p^{\leqslant R}=\underset{p \in C^{*}}{\arg \min } f_{y}(p): \underset{p \in C^{*}}{\arg \min } f_{x}(p) \\
p_{y}^{r e f}-f_{y}(p)+f_{x}(p)-p_{x}^{r e f} \leqslant R \\
f_{x}(p)<p_{x}^{>R}
\end{gathered}
$$

Eq. 7 constrains the resulting solution $p^{\leqslant R}$ to be within the range of $p^{r e f}$ w.r.t. the radius $R$, while Eq. 8 ensures the solution is not dominated by $p^{\leqslant R}$.

Note that minimising $f_{x}$ or $f_{y}$ within or outside of radius $R$ is not enough to guarantee Pareto optimality. As previously discussed, the Pareto optimal solution $p^{>R}$, which is the closest Pareto optimal solutions to $p^{r}$ ef that is outside of radius $R$, is used in the computation of $p^{\leqslant R}$, the further Pareto optimal solution from $p^{r e f}$ (with greater $f_{x}$ ) within radius $R$. To see that importance of the solution $p^{>} R$, consider a bi-optimisation problem that implicitly defines points $(2,4),(3,3),(4,2),(4,0)$ and let $p^{r e f}=(2,4)$ and $R=4$. The solution $p^{\leqslant R}=(3,3)$, but merely minimising $f_{y}$ within 
radius $R$ yields $(4,2)$, which is not correct since it is dominated by $p^{>R}=(4,0)$. However, to compute $p^{>R}$ we need ybound (Eq. 1-2), i.e., the minimum $f_{y}$ within the radius. Consider another example with $(2,4),(3,1),(4,1),(5,0)$ and $p^{r e f}=(2,4)$ and $R=4$. Minimising $f_{x}$ outside radius $R$ produces $(4,1)$, which is dominated by $(3,1)$, but the correct solution $p^{>R}$ is $(5,0)$. Once ybound is enforced, $p^{>R}=(5,0)$ is correctly computed.

As in the explicit $f w r$ case, the method $f w r 2$ is guaranteed to produce a solutions since the reference solution $p^{\text {ref }}$ can be returned.

Helper method filter $2\left(p^{r e f},\left\langle X, C, f_{x}, f_{y}\right\rangle, R\right)$ : Given a Pareto optimal solution $p^{r e f}$, a bi-objective optimisation problem $\left\langle X, C, f_{x}, f_{y}\right\rangle$, and a radius $R$, the task is to solve a restriction $\left\langle X, C^{\prime}, f_{x}, f_{y}\right\rangle$ of the problem, i.e. $C \subseteq C^{\prime}$, so that to discard the Pareto optimal solutions from $C^{*}$ that are within the range of $p^{r e f}$ w.r.t. the radius $R$. The updated set $C^{\prime}$ of constraints is defined as $C^{\prime}=C \cup c_{1} \cup c_{2}$, where $c_{1}$, $c_{2}$ are defined as follows:

$$
\begin{gathered}
c_{1}: p_{y}^{r e f}-f_{y}(p)+f_{x}(p)-p_{x}^{r e f}>R \\
p^{\leqslant R}=f w r 2\left(p^{r e f},\left\langle X, C, f_{x}, f_{y}\right\rangle, R\right) \\
c_{2}: f_{y}(p)<p_{y}^{\leqslant R}
\end{gathered}
$$

Eq. 11 enforces that the solutions are not dominated by $p^{\leqslant R}$, while Eq. 9 ensures that the solutions are outside the range of $p^{r e f}$ w.r.t. the radius $R$.

Helper method $p 2_{\min }^{x}\left(\left\langle X, C, f_{x}, f_{y}\right\rangle\right)$ : Given a biobjective optimisation problem $\left\langle X, C, f_{x}, f_{y}\right\rangle$, the task is to compute the Pareto optimal solution $p 2_{\min }^{x}$ with a minimum value for $f_{x}$. This is done by a lexicographical problem:

$$
p 2_{\text {min }}^{x}=\underset{p \in C^{*}}{\arg \min } f_{x}(p): \underset{p \in C^{*}}{\arg \min } f_{y}(p)
$$

The procedure $R S-R 2\left(\left\langle X, C, f_{x}, f_{y}\right\rangle, R\right)$, which computes the representative set given a bi-objective optimisation problem $\left\langle X, C, f_{x}, f_{y}\right\rangle$, can be analogously defined as in Algorithm 1 using the helper methods defined in this section. In a similar fashion, $\operatorname{Rep} \operatorname{Set} 2(P F, k)$ can be derived from Algorithm 2 by using $R S-R 2$. Note that none of the steps in the algorithm requires to compute the Pareto front $P F$ of the bi-objective optimisation problem $\left\langle X, C, f_{x}, f_{y}\right\rangle$, but the resulting output is the $k$-representative set.

Proposition 5 The procedure RepSet $2\left(\left\langle X, C, f_{x}, f_{y}\right\rangle, k\right)$ computes a $k$-representative set by using $\mathcal{O}\left(k \cdot \log \left(R_{U B}\right)\right)$ solver calls, where $R_{U B}=\left\lfloor\frac{\operatorname{dist}\left(p_{\min }^{x}, p_{\max }^{x}\right)}{k}\right\rfloor$.

Note that $R_{U B}$ represents a value that is exponential in the input size, but the overall complexity remains polynomial as it relies on $\log \left(R_{U B}\right)$. The proof of the proposition is analogous to Prop. 2, but individual elements of the Pareto front are computed using solver calls rather than accessed in constant time.
An interesting consequence of Prop. 5 is that focussing on the bi-objective case to compute representative solutions leads to a computational shift in comparison to the general multi-objective case. Indeed, let us consider the decision problem related to the computation of representative solutions introduced in (Schwind et al. 2016):

Definition 2 (DP2 (Schwind et al. 2016)) Given a multiobjective optimisation problem $P=\langle X, C, F\rangle$ where $F$ is a set of objective functions, and two integers $\alpha, k$ where $k$ is bounded by a polynomial in the size of $P$, does there exist $S \subseteq P F$ such that $|S|=k$ and $\Omega(S) \leqslant \alpha$ ?

Proposition 6 ((Schwind et al. 2016)) DP2 is NPNP -hard.

Proposition 7 If $P=\langle X, C, F\rangle$ and $F$ consists of at most two objective functions, DP2 is in $\mathrm{P}^{\mathrm{NP}}$.

This result shows that, even though there may be an exponential number of Pareto optimal solutions, computing the representative set only requires at most a polynomial number of solver calls, regardless of the size of the Pareto front. Thus, computing a representative set is done more efficiently than enumerating the Pareto front. This also shows that for linear bi-objective programs, representative solutions can be computed in polynomial time despite infinite-sized Pareto fronts. To the best of our knowledge, this is the first result of this kind for bi-objective optimisation, as previous works compute either an approximate set or the complete Pareto front. We illustrate the practicality of our algorithm in the next section.

\section{Experimental Results}

We implemented our algorithms and performed a numerical study. The goal was to verify the theoretical result and evaluate the empirical performance. The computational study has a supporting role and is meant as a proof-of-concept rather than a rigorous comparison of solving paradigms and techniques, which is out of the scope of the paper. To the best of our knowledge, this is the first time a theoretical result (Prop. 5 ) and algorithm have been provided for computing provably optimal representative solutions without resorting to a complete Pareto front enumeration. Possibly better results could be achieved with further algorithmic improvements, in particular by exploiting problem-specific features, but this does not demean our main results: a theoretical result and principled algorithms to compute representative solutions for biobjective optimisation.

Our code and benchmarks are available online: bitbucket.org/EmirD/representative-solutions-for-bi-

objective-optimisation. The input format for our program is an MPS file. MiniZinc (Nethercote et al. 2007) users can convert MZN/DZN files to the MPS format using the built-in conversion feature of MiniZinc.

\section{Computational Setting and Benchmarks}

Experiments were performed on a machine with an i7$7700 \mathrm{HQ}$ CPU @ 2.80GHz processor and 32 GB of RAM, running one instance at a time with a time limit of ten hours. We used Gurobi as the optimisation solver and experimented with two benchmark families with varying instance size: 
- Resource-constrained project scheduling problems with weighted earliness and tardiness objectives, labelled as RCPSP-wet in the MiniZinc Challenge 2016 and 2017. The instances are naturally bi-objective as the objective consists of two parts: earliness and tardiness. For ease of presentation we partitioned the benchmarks in three groups: RCPSP-30, -60, and -90, containing six small, three medium, and three large instances. We excluded the large j90-10 benchmark as none of the techniques could produce meaningful results within ten hours.

- Generated large bi-objective set covering benchmarks. Similar instances were used in other single- and multiobjective works (Musliu 2006; Bergman and Cire 2016). The characteristics of the benchmarks are as follows: the number of columns $n=\{1000,2000\}$, number of rows $\frac{n}{5}$, each row can be covered by ten $(n=1000)$ or twenty $(n=2000)$ randomly selected columns, and each column has a random weight from the interval $[1,1000]$ for each objective. The task is to cover each row while minimising the cost of the selected columns as a bi-objective problem. We created twenty benchmarks, ten for each value of $n$.

In the following, we consider the implicit Pareto front case. For the explicit case, the k-representative solutions can be computed in seconds, as the dominating complexity factor is sorting, and is thus not further considered.

\section{Results and Discussion}

We compare our approach with two techniques that compute the Pareto front: the $\epsilon$-method (Haimes 1971) and the two-phase approach (Ulungu and Teghem 1995). Although somewhat old, these techniques are the two most widely used generic methods for Pareto front enumeration in biobjective optimisation for integer programming according to a recent survey (Stidsen, Andersen, and Dammann 2014). As the time required to compute a representative set given a Pareto front is negligible for bi-objective problems, we compare against generating the complete Pareto front. In further text, we discuss the results in comparison to the $\epsilon$-method since it was an order-of-magnitude faster than the two-phase approach in our experiments. The discrepancy is likely because we used a generic implementation of the second phase in the two-phase approach, which is typically tailored to particular applications. We would like to note that we are not aware of other techniques that directly compute the representative set: this is our main contribution.

The results are given in Table 1 . We consider values $[1,2,3,4,5]$ for $k$, the number of desired representative solutions. There is a correlation between $k$ and the runtime of our approach. Similarly, the number of iterations are kept low (recall that each iteration solves a decision problem). These observations are in accordance with the computational complexity (Prop. 5).

Our method uses a fewer number of solver calls compared to Pareto front enumeration for the considered benchmarks. This results in better performance. The conclusion holds irrespective of $k$, the size of the representative set. The gain in time is roughly proportional to the difference in the number of solver calls. Furthermore, the number of intermediate so-

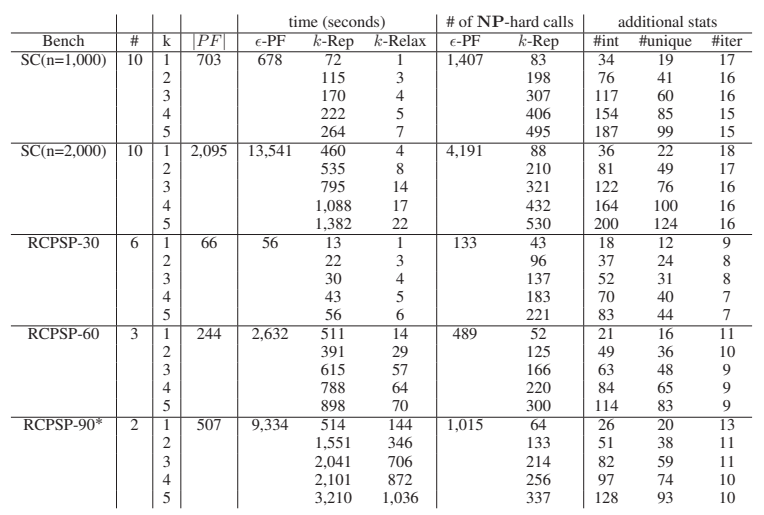

Table 1: Comparison of the $\epsilon$-method and our $k$ representative approach. The column ' \#' shows the number of benchmarks; $|P F|$ is the size of the PF; $k$-Relax refers to the k-representative solution of the linear relaxation; \#int and \#unique is the number of intermediate and unique solutions found during the search; \#iter denotes the number of iterations. Results are averaged. *The Pareto front could not be generated for one benchmark (j-90-19) within ten hours, but we could compute the representative solutions.

lutions generated throughout the search is typically considerably smaller than the number of Pareto optimal solutions. These results are in line with our main claim, i.e., computing the representative solutions can be done efficiently without resorting to the complete Pareto front.

During the search, the same Pareto optimal solution can be computed more than once, i.e., the number of unique solutions is smaller than the number of intermediate solutions. This indicates an overlap between the iterations.

We considered the linear relaxation of the benchmarks, i.e. the integrality constraints of the variables are relaxated to continous variables. In these instances, the Pareto front is of infinite size, but our approach can compute the $k$ representative set. The lower run time for the relaxed instances is expected, given that in general linear programs are easier to solve than integer programs.

\section{Conclusion}

We provide a novel algorithm for computing the representative solutions for bi-objective optimisation. We show an improved complexity result over the general multi-objective case. Moreover, the numerical study illustrates the practicality of our approach. For future work, we would reduce potential redundancy in subsequent iterations of our algorithm by taking into account previously computed solutions, and incorporate other properties of Pareto optimal solutions, such as those proposed in (Bazgan, Jamain, and Vanderpooten 2017), to further refine the representative set.

\section{References}

Adomavicius, G., and Kwon, Y. 2014. Optimization-based approaches for maximizing aggregate recommendation diversity. INFORMS Journal on Computing 26(2):351-369. 
Bazgan, C.; Jamain, F.; and Vanderpooten, D. 2017. Discrete representation of the non-dominated set for multi-objective optimization problems using kernels. European Journal of Operational Research 260(3):814-827.

Bergman, D., and Cire, A. A. 2016. Multiobjective optimization by decision diagrams. In Proc. of the 22nd International Conference on Principles and Practice of Constraint Programming (CP'16), 86-95. Springer.

Caillouet, C.; Li, X.; and Razafindralambo, T. 2011. A multi-objective approach for data collection in wireless sensor networks. In Proc. of the 10th International Conference on Ad-hoc, Mobile, and Wireless Networks (ADHOCNOW'11), 220-233.

Ceyhan, G.; Köksalan, M.; and Lokman, B. 2019. Finding a representative nondominated set for multi-objective mixed integer programs. European Journal of Operational Research 272(1):61-77.

Dhar, R. 1997. Consumer Preference for a No-Choice Option. Journal of Consumer Research 24(2):215-231.

Eusébio, A.; Figueira, J.; and Ehrgott, M. 2014. On finding representative non-dominated points for bi-objective integer network flow problems. Computers and Operations Research 48:1-10.

Gavanelli, M. 2002. An algorithm for multi-criteria optimization in CSPs. In Proc. of the 15th European Conference on Artificial Intelligence (ECAI'02), volume 2, 136-140.

Haimes, Y. 1971. On a bicriterion formulation of the problems of integrated system identification and system optimization. IEEE transactions on systems, man, and cybernetics 1(3):296-297.

Hebrard, E.; Hnich, B.; O'Sullivan, B.; and Walsh, T. 2005. Finding diverse and similar solutions in constraint programming. In Proc. of the 20th National Conference on Artificial Intelligence (AAAI'05), 372-377.

Iyengar, S. S., and Lepper, M. R. 2000. When choice is demotivating: Can one desire too much of a good thing? Journal of personality and social psychology 79(6):995.

Karasakal, E., and Köksalan, M. 2009. Generating a representative subset of the nondominated frontier in multiple criteria decision making. Operations research 57(1):187199.

Kwek, S., and Mehlhorn, K. 2003. Optimal search for rationals. Information Processing Letters 86(1):23-26.

Masin, M., and Bukchin, Y. 2008. Diversity maximization approach for multiobjective optimization. Journal of Operations Research 56(2):411-424.

Musliu, N. 2006. Local search algorithm for unicost set covering problem. In Proc. of the 19th International Conference on Industrial, Engineering and Other Applications of Applied Intelligent Systems (IEA/AIE)'06, 302-311.

Nethercote, N.; Stuckey, P. J.; Becket, R.; Brand, S.; Duck, G. J.; and Tack, G. 2007. Minizinc: Towards a standard $\mathrm{cp}$ modelling language. In Proc. of the 13th International Conference on Principles and Practice of Constraint Programming (CP'07), 529-543. Springer.
Papadimitriou, C. H. 2003. Computational complexity. John Wiley and Sons Ltd.

Petit, T., and Trapp, A. C. 2015. Finding diverse solutions of high quality to constraint optimization problems. In Proc. of the 24th International Joint Conference on Artificial Intelligence (IJCAI'15), 260-267.

Petit, T., and Trapp, A. C. 2019. Enriching solutions to combinatorial problems via solution engineering. INFORMS Journal on Computing 31(3):429-444.

Raith, A., and Sedeño-Noda, A. 2017. Finding extreme supported solutions of biobjective network flow problems: An enhanced parametric programming approach. Computers and Operations Research 82:153-166.

Sayin, S. 2000. Measuring the quality of discrete representations of efficient sets in multiple objective mathematical programming. Mathematical Programming 87:543-560.

Schaus, P., and Hartert, R. 2013. Multi-objective large neighborhood search. In Proc. of the 19th International Conference on Principles and Practice of Constraint Programming (CP'13), 611-627.

Schwind, N.; Okimoto, T.; Clement, M.; and Inoue, K. 2016. Representative solutions for multi-objective constraint optimization problems. In Proc. of the 15th International Conference of Principles of Knowledge Representation and Reasoning (KR'16), 601-604.

Shafir, E.; Simonson, I.; and Tversky, A. 1993. Reasonbased choice. Cognition 49(1-2):11-36.

Stidsen, T.; Andersen, K. A.; and Dammann, B. 2014. A branch and bound algorithm for a class of biobjective mixed integer programs. Management Science 60(4):1009-1032.

Trisna, T.; Marimin, M.; Arkeman, Y.; and Sunarti, T. 2016. Multi-objective optimization for supply chain management problem: A literature review. Decision Science Letters 5(2):283-316.

Ulungu, E. L., and Teghem, J. 1995. The two phases method: An efficient procedure to solve bi-objective combinatorial optimization problems. Foundations of Computing and Decision Sciences 20(2):149-165.

Vaz, D.; Paquete, L.; Fonseca, C. M.; Klamroth, K.; and Stiglmayr, M. 2015. Representation of the non-dominated set in biobjective discrete optimization. Computers and $O p$ erations Research 63:172-186.

Zitzler, E.; Thiele, L.; Laumanns, M.; Fonseca, C. M.; and Da Fonseca, V. G. 2003. Performance assessment of multiobjective optimizers: An analysis and review. IEEE Transactions on evolutionary computation 7(2):117-132. 